\title{
Comparing the short and ultrashort pulsed laser ablation of LiF
}

\author{
Simon J. Henley a) \\ Advanced Technology Institute, School of Electronics and Physical Sciences, University of Surrey, \\ Guildford, GU2 7XH, United Kingdom
}

Gareth M. Fuge and Michael N. R. Ashfold

School of Chemistry, University of Bristol, Bristol, BS8 1TS, United Kingdom

(Received 26 July 2004; accepted 11 October 2004; published online 23 December 2004)

\begin{abstract}
Pulsed laser ablation of LiF was studied using both nanosecond (ns) and femtosecond (fs) pulses at $248 \mathrm{~nm}$. Optical emission from electronically excited Li and F atoms in the plume of ejected material was investigated by wavelength, time and spatially resolved imaging methods. Careful analysis of images of species selected optical emission yielded estimates of the mean velocities of the $\mathrm{Li}^{+}$ions arising in both excitation schemes $(\sim 11$ and $\sim 13 \mathrm{~km} / \mathrm{s}$, respectively), and highlighted the dramatic effects of radiation trapping, most notably by the reabsorption of $\operatorname{Li}(2 p \rightarrow 2 s)$ emission by ground state Li atoms in the ns ablation studies. Plumes formed by fs excitation are found to contain a higher fraction of energetic/electrically excited components, including excited $\mathrm{F}$ atoms and ions, indicative of an explosive boiling mechanism, whereas the ablation plume resulting from ns ablation is deduced to arise primarily from thermal evaporation of the transiently heated target surface. The amount of target material removed per shot is significantly less in the case of fs excitation. The density (and size) of unwanted droplets in films grown by fs ablation is much smaller than in the case of ns ablation, especially on substrates mounted in an off-axis ablation geometry, implying that hydrodynamic sputtering is much reduced by the use of short pulses and that fs ablation must be the preferred route to forming very thin LiF coatings. (C) 2005 American Institute of Physics. [DOI: 10.1063/1.1828241]
\end{abstract}

\section{INTRODUCTION}

Pulsed laser ablation (PLA), as a film deposition technique and as a chemical analysis tool, has greatly matured over the last decade. However, a detailed understanding of the ablation process has proved elusive due to the plethora of mechanisms for the removal of target material, the complex nature of the initial laser-target interaction, and the dynamic nature of the interaction between the plume and the laser pulse. $^{1-3}$ This complexity is further increased when considering the ablation of materials that are nominally transparent to the laser photons.

Traditionally PLA has been performed using lasers giving nanosecond duration pulses. However, the advent of subpicosecond pulsed lasers opened the possibility of decoupling the initial photoexcitation of the target material from the subsequent dynamics of the removed atomic, ionic, and molecular plume components. This decoupling removes a layer of complexity from the ablation process, thereby facilitating the investigation and understanding of PLA mechanisms. Ultrashort pulsed lasers offer dramatically higher peak powers than nanosecond lasers. Multiphoton pathways for ablation become more significant at higher incident intensities. This is of particular importance when considering the ablation of substances with band gaps larger than the energy of the laser photons employed-as, for example, will generally be the case with the alkali halides.

Production of thin coatings of alkali halides, particularly

\footnotetext{
a) Author to whom correspondence should be addressed; electronic mail: s.henley@eim.surrey.ac.uk
}

$\mathrm{LiF}$, is of significant interest for a variety of reasons. Thin $\mathrm{LiF}$ coatings have been demonstrated to reduce the work function of metal electrodes, ${ }^{4}$ permitting more efficient electron injection and field emission. Alkali halide coatings can act as effective dipole layers, inducing a negative electron affinity in diamond surfaces by lowering the surface work function. ${ }^{5} \mathrm{KCl}$ and $\mathrm{KI}$ coatings have recently been shown to increase the photoluminescence of $\mathrm{ZnO}$ crystals by up to 300 times. ${ }^{6}$ The enhanced electron injection at LiF coated cathodes has also been used to improve the performance of organic electroluminescence devices. ${ }^{7,8}$

PLA is recognized as a versatile and useful technique for the deposition of very thin coatings, but suffers due to the production of unwanted asperities such as droplets and macroparticles_-particularly when applied to the ablation of ultrawide band gap materials, such as LiF. ${ }^{9,10}$ For example, a $\mathrm{KrF}$ excimer laser photon has an energy of $5 \mathrm{eV}$, while the band gap of $\mathrm{LiF}$ is in the range $13.6-14.5 \mathrm{eV} .{ }^{11,12}$ Electronic sputtering processes would be expected to be the dominant material ejection mechanism in such a regime, since bandto-band excitation would require a MP absorption process. ${ }^{13}$

There have been relatively few PLA studies of alkali halides, ${ }^{9,10,14-16}$ particularly involving ultrashort pulse ablation. ${ }^{17-19}$ Several studies of laser interaction with these materials have concentrated on the subablation threshold photodesorption of ionic species from single crystal surfaces, ${ }^{17,18,20,21}$ and have typically employed mass spectroscopy to analyze components in the plume of ejected gas phase material. In this article we compare the $248 \mathrm{~nm}$ PLA of $\mathrm{LiF}$ using nanosecond and subpicosecond pulses. The 
temporal and spatial evolution of the optical emission from the plume is investigated using time-gated imaging and the ablation mechanisms, including the production of droplets, are studied.

\section{EXPERIMENTAL DETAILS}

The ablation apparatus consisted of a stainless steel vacuum chamber which was evacuated using a turbomolecular pump, backed by a rotary pump to a base pressure of $1 \times 10^{-6}$ Torr. The chamber was equipped with several sidearms, each of which was sealed with a quartz window, permitting wide spectral-range optical observation of the plume produced during ablation. The incident laser beam propagated through one of the sidearms, in the $x y$ plane at $45^{\circ}$ to the target surface normal-here defined as the $x$ axis. The viewing port lay in the $x y$ plane also and allowed optical emission accompanying the ablation plume to be monitored by viewing along the $y$ axis (i.e., parallel to the target surface). The target, a 2-in.-diam sintered disk of $99.9 \%$ pure $\mathrm{LiF}$ (Testbourne, UK), was rotated during the experiments in order to avoid repeated ablation of the same spot on the target. Subpicosecond $248 \mathrm{~nm}$ laser pulses were generated using a $\mathrm{XeCl}$ excimer pumped dye laser system producing $450 \mathrm{fs}$ pulses from a distributed feedback dye laser at $496 \mathrm{~nm}$ which, after frequency doubling, were amplified in the $\mathrm{KrF}$ excimer laser cavity. ${ }^{22,23}$ The KrF cavity (LambdaPhysik EMG150MSC), operating unseeded, provided pulses of $15 \mathrm{~ns}$ full width at half maximum at $248 \mathrm{~nm}$. The outputs of the nanosecond excimer laser and the frequency doubled dye laser had markedly different spatial profiles, beam divergences, and peak powers, which meant that these two sources produced very different spot sizes when focused at the target. Typical laser pulse energies (and fluences) employed in the experiments described here were $\sim 47 \mathrm{~mJ} /$ pulse $\left(12.5 \mathrm{~J} / \mathrm{cm}^{2}\right)$ in the case of nanosecond $(\mathrm{ns})$ ablation and $\sim 8 \mathrm{~mJ} /$ pulse $\left(12.3 \mathrm{~J} / \mathrm{cm}^{2}\right)$ for the femtosecond (fs) studies. Although the fluences used were similar, the power density was nearly five orders of magnitude lower for the ns, as compared with the fs, pulses.

The optical emission accompanying the ablation plume was collected in two different ways. To record spatially resolved optical emission spectra (OES) the emission was focused (in a 1:1 magnification configuration) onto a quartz fiber directed so as to view a column along the $y$ axis. The exit of the fiber bundle abutted the entrance slit (width set at $30 \mu \mathrm{m}$ ) of a $0.32 \mathrm{~m}$ spectrograph (TRIAX-320, Jobin Yvon/ Spex) equipped with three, user selectable, holographic gratings, with 600, 1800, and 2400 lines/mm, providing spectral resolutions of $0.4,0.15$, and $0.1 \mathrm{~nm}$, respectively. The resulting dispersed emission could be recorded over a wavelength range 360-900 nm, using an intensified charge coupled device (i-CCD DH520-18F, Andor Technology.) By translating the position of the entrance of the fiber, the variation of the OES along the $x$ axis of the plume could be measured. The fiber had a diameter of $0.6 \mathrm{~mm}$, which defined the spatial resolution. The second method of recording the plume emission involved imaging the evolving spatial distributions of selected emitting species by using the i-CCD camera in time- gated detection mode, decoupling it from the spectrometer, and focusing the optical emission (either total emission, or just the fraction transmitted through a suitably chosen narrow bandpass interference filter) onto the $1024 \times 256$ diode array. The spatial calibration of the i-CCD image was achieved by taking an image of a rule illuminated by a white light source. The minimum delay, after which an i-CCD image of the plume could be taken, was limited to 120 ns by the response time of the electronics used.

Droplet production during the $\mathrm{ns}$ and fs ablation of $\mathrm{LiF}$ was investigated by placing a number of silicon substrates at different angles to the target normal, but at the same radial distance $(6 \mathrm{~cm})$ from the ablation spot on the target. A similar off-axis ablation geometry was used elsewhere in studies of the ns PLA of LiF at $193 \mathrm{~nm} .{ }^{10}$ The droplet density was ascertained from scanning electron microscope (SEM) images obtained in a Philips S4000 field emission SEM operating at $20 \mathrm{kV}$.

\section{RESULTS}

\section{A. Effect of pulse duration on film deposition}

The ns ablation of $\mathrm{LiF}$ at $193 \mathrm{~nm}$ has been shown to produce large numbers of molten droplets by hydrodynamic sputtering. ${ }^{9,10}$ This is thought to be a consequence of the large penetration depth of such laser photons into $\mathrm{LiF}$, coupled with the relatively low melting point of the material $\left(848^{\circ} \mathrm{C}\right)^{24}$ and its high coefficient of thermal expansion $\left(37 \times 10^{6} \mathrm{~K}^{-1}\right) .{ }^{24}$ During the ns pulse, a sizable depth of material (estimated as $\sim 1 \mu \mathrm{m}$ for $193 \mathrm{~nm}$ ablation ${ }^{10}$ ) expands rapidly as it is heated and liquefies. When the deeper regions of the molten irradiated area solidify and contract, liquid droplets of the surface can be ejected. ${ }^{13}$

The initial temperature rise within the irradiated region can be expected to be much more rapid in the case of fs ablation, since the energy is delivered over a time scale that is short compared to the rate of phonon-phonon coupling. In this regime, material will be vaporized more quickly, reducing the extent of hydrodynamic sputtering. In order to compare the production of droplets from ns and fs ablation, films were deposited on substrates mounted at a range of different angles to the $x$ axis. Figure 1 shows SEM images of the films produced by LiF ablation using $500 \mathrm{~ns}$ laser pulses [left-hand side, images (a)-(d)] and one thousand fs pulses [right-hand side, images (e)-(h)]. The substrates were placed at angles to the target normal of (a) and (e) $0^{\circ}$, (b) and (f) $15^{\circ}$, (c) and (g) $45^{\circ}$ and (d) and (h) $60^{\circ}$. A high density of asperities, characterized as splashed droplets, are observed in the films grown using both ns and fs laser pulses, but the droplet density is seen to decrease as the deposition angle was increased. Comparing films deposited with ns and fs pulses we see that, at all angles $\theta$, the size and density of droplets is much reduced in the case of fs PLA, especially when it is recalled that twice as many pulses were used in the production of these films.

Figure 2 shows a plot of the droplet density per ns (upper curve) and fs (lower curve) laser shot obtained from analysis of a range of images similar to those shown in Fig. 1. Image processing was carried out using commercial image 


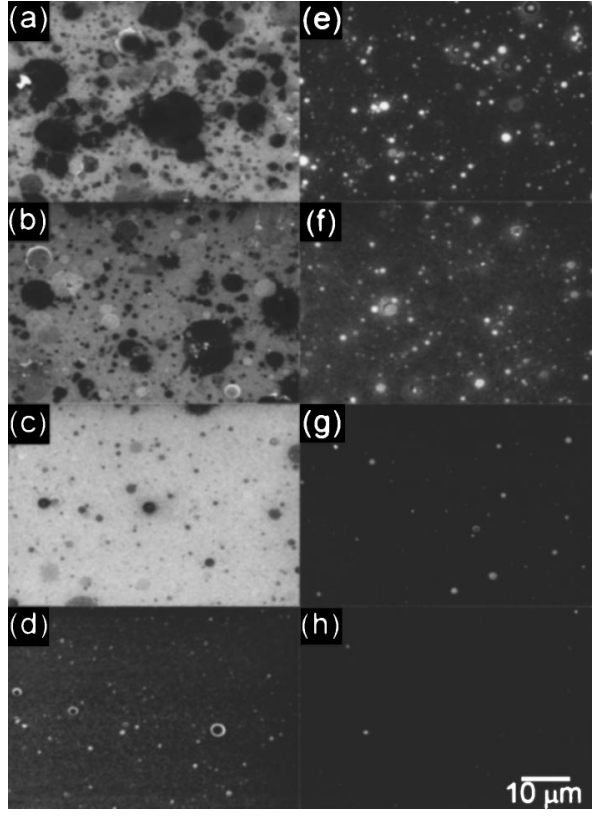

FIG. 1. SEM images of films and accompanying droplets produced by the ns [left-hand side, images (a)-(d)] and fs [right-hand side, images (e)-(h)] PLA of $\mathrm{LiF}$ at $248 \mathrm{~nm}$. The former were obtained using 500 laser shots, while the latter were the result of $1000 \mathrm{fs}$ laser pulses. The substrates were placed at angles to the target normal of (a) and (e) $0^{\circ}$, (b) and (f) $15^{\circ}$, (c) and (g) $45^{\circ}$ and (d) and (h) $60^{\circ}$.

analysis software $^{25}$ (the minimum size object that was considered to be a droplet was chosen to be $80 \mathrm{~nm}$ ). The error bars represent the standard error on the mean droplet density taken from three images from different areas on the same sample. The line fitted to the fs data gives an angular dependence of the droplet density of approximately $\cos ^{2.6}(\theta)$. No sensible fitting of the nanosecond droplet density was possible as the larger drops, at the higher densities, masked the underlying distribution of smaller droplets. This can be seen most dramatically in Fig. 1(a), where the largest droplets (which appear to have struck the substrate while still molten, and have very low aspect ratios) take up a considerable fraction of the surface area. Clearly, for both ns and fs ablation, the droplet density is much reduced by growing in an offaxis geometry. However, these images give little information about the relative film thickness as a function of pulse dura-

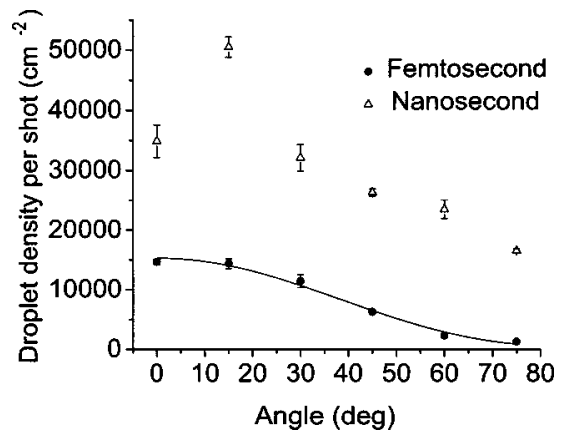

FIG. 2. Plot of the droplet density per laser shot for a range of substrates, placed at the same distance from the ablation spot, but at different angles to the target surface normal. The upper and lower curves are for ns and fs ablation, respectively. The error bars represent the standard error on the mean of three different areas on the same sample.

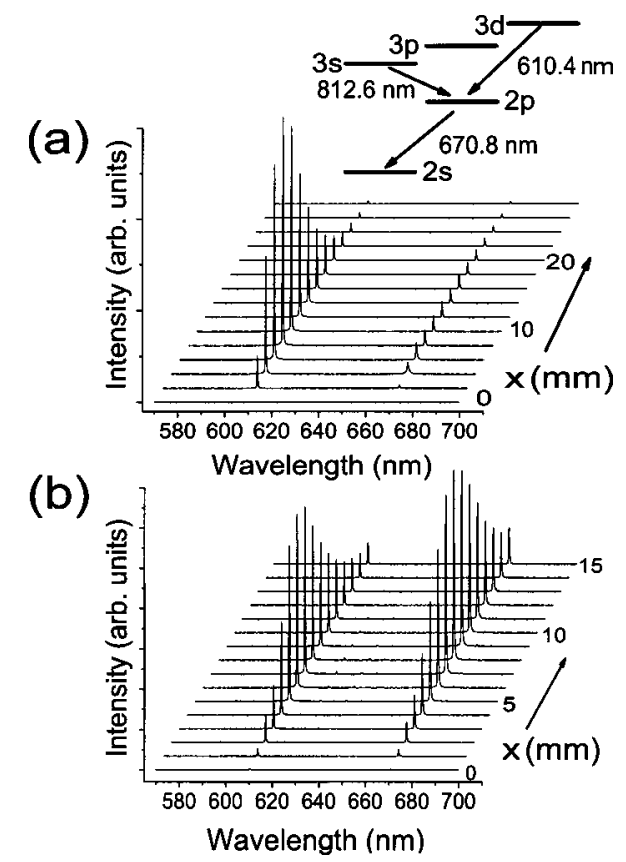

FIG. 3. Wavelength resolved OES spectra recorded at different distances $x$ from the laser spot for (a) $\mathrm{ns}$ and (b) fs PLA of LiF at $248 \mathrm{~nm}$ by capturing emission in a $1 \mu$ s time gate delayed by $t 120 \mathrm{~ns}$ after the start of laser excitation. These spectra only show the wavelength region around the two main Li I spectral lines. The schematic energy level diagram inset in (a) shows the transitions responsible for these two emissions.

tion and the angle at which the substrate is mounted. If the droplet density is less at higher angles, but the film thickness is reduced by a greater factor, the volume fraction of particulates will actually be increased. Even if this was the case, however, the droplet size is much reduced by depositing at higher angles, so the film quality is likely to be improved. We note that the contrast in the SEM images shown in Figs. 1(a)-1(c) appears different from the other images, including those of all films deposited with fs pulses. The background in images (a)-(c) shows up brighter than the droplets, whereas this contrast is reversed for the other films. Such an observation is characteristic of surface charging, and implies that the (insulating $\mathrm{LiF}$ ) film thickness is significantly larger in the case of films deposited with ns pulses.

\section{B. Spatially resolved optical emission spectroscopy}

Excited species in the plume were investigated by monitoring OES spectra at different positions along the $x$ axis. Figure 3 shows OES spectra covering the wavelength range 570-700 nm taken at different distances from the lasertarget interaction region using (a) ns and (b) fs pulses. Signal was collected in a $1 \mu$ s gate on the spectrometer, which opens at $t=120 \mathrm{~ns}$ after the laser pulse is incident on the target. This spectral region is dominated by the two main Li I spectral lines. The relevant portion of the Grotrain diagram of neutral Li is shown above (a). Other Li I emissions were observed in other spectral regions of the spectrum, most notably the $3 s \rightarrow 2 p$ transition at $812.6 \mathrm{~nm}$ (also shown in the inset), but all were much weaker than the 610.4 and $670.8 \mathrm{~nm}$ emissions (henceforth termed the $\mathrm{Li}$ a and $\mathrm{Li} \mathrm{b}$ emissions, respectively). As Fig. 3 shows, the relative inten- 


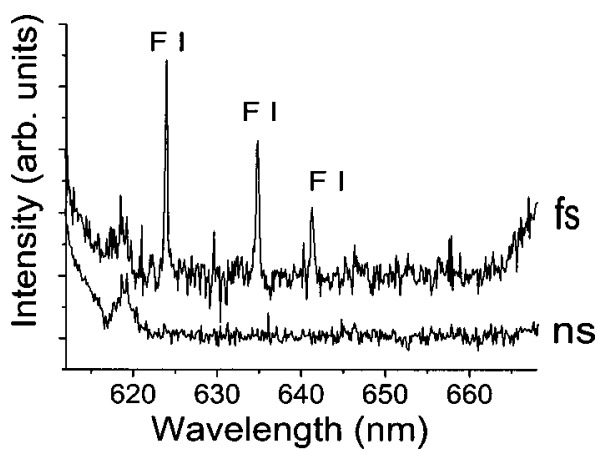

FIG. 4. Wavelength resolved OES spectra recorded at $x=7 \mathrm{~mm}$ following ns (lower spectrum) and fs (upper spectrum) PLA of LiF at $248 \mathrm{~nm}$. F I emission lines are only observed at this distance in the fs PLA study.

sities of these two peaks are sensitively dependent on the laser pulse duration used. $\mathrm{Li}$ a emission dominates the OES spectrum accompanying ns PLA, and the ratio of the $\mathrm{Li}$ a to $\mathrm{Li} b$ emission intensities is greater for ns PLA than for fs PLA at all $x$. This is at first sight surprising, given that the emitting (3d) states responsible for $\mathrm{Li}$ a emission lie higher in energy. The explanation for these differences lies in the fact that the Li b emission terminates at the ground $(2 s)$ state, and so can be reabsorbed if there is a significant population of ground state neutral Li atoms. As the asymptotic fate of all gas phase $\mathrm{Li}$ atoms is the ground electronic state, a significant population of $\mathrm{Li}(2 s)$ atoms is to be expected. Inspection of the ( $\mathrm{Li} \mathrm{a})$ :(Li b) intensity ratio in Figs. 3(a) and 3(b) leads to the conclusion that self-absorption of the Li b emission is much more important in the case of ns ablation than in fs ablation plume-which appears optically thin in comparison. Such accords with the conclusions drawn from analysis of the deposited films, which indicate a much greater material removal per pulse in the case of ns PLA. This aspect is investigated further below, using plume imaging techniques, and will be discussed later.

OES spectra recorded following fs ablation [Fig. 3(b)] show additional weak lines between the $\mathrm{Li}$ a and $\mathrm{Li} \mathrm{b}$ transitions. Figure 4 shows a magnified section of OES spectra taken $7 \mathrm{~mm}$ from the laser interaction volume for both $\mathrm{ns}$ (lower trace) and fs (upper spectrum) PLA. Three additional lines are clearly evident, at 624.0, 634.9, and $641.4 \mathrm{~nm}$, in the case of fs laser excitation only. All three are attributable to $3 p \rightarrow 3 s$ transitions in atomic fluorine, and originate from an excited state that lies $14.68 \mathrm{eV}$ above the ground state. ${ }^{26}$

\section{Time resolved imaging of specific plume emissions}

Before discussing the temporal evolution of the optical emission from the plume, it is important to reflect on the time scales involved. The radiative lifetimes (i.e., the times during which an initial excited state population will decay by a factor of $e^{-1}$ under collision-free conditions) of excited states responsible for the stronger Li I emissions are shown in Table I. However, in the following results, it will be shown that Li I emissions are still readily detectable after $>10$ lifetimes, by which time the spontaneous emission intensities should have dropped by a factor of $10^{4}$.
TABLE I. The radiative lifetimes (under collision-free conditions) of excited states responsible for the stronger Li I emissions.

\begin{tabular}{ccc}
\hline \hline $\begin{array}{c}\text { Emission } \\
\text { wavelength }(\mathrm{nm})\end{array}$ & $\begin{array}{c}\text { Electronic } \\
\text { transition }\end{array}$ & $\begin{array}{c}\text { Radiative } \\
\text { lifetime (ns) }\end{array}$ \\
\hline 812.6 & $1 s^{2} 3 s \rightarrow 1 s^{2} 2 p$ & 29 \\
610.4 & $1 s^{2} 3 d \rightarrow 1 s^{2} 2 p$ & 14 \\
670.8 & $1 s^{2} 2 p \rightarrow 1 s^{2} 2 s$ & 27 \\
\hline
\end{tabular}

A mechanism for populating the observed emitting levels after the ablation event is required in order to explain the duration of the observed emissions. Recombination of $\mathrm{Li}^{+}$ ions and electrons, stabilized by a third body, (or $\mathrm{Li}^{+}$neutralization by electron transfer from $\mathrm{F}^{-}$ions) constitutes one obvious source of excited neutral $\mathrm{Li}$ atoms. As suggested previously, ${ }^{27}$ such electron ion recombination (EIR) is likely to proceed via highly excited Rydberg states, and subsequent radiative (and/or collisional) cascade via the monitored transitions and, ultimately, to the ground electronic state, as shown in Eq. (1)

$$
\begin{aligned}
\mathrm{Li}^{+}+\mathrm{e} & \rightarrow \mathrm{Li}^{*} *(\text { high } n, \ell, m) \\
& \rightarrow \rightarrow \rightarrow \mathrm{Li} * *(3 d \text { or } 3 s) \\
& \rightarrow \mathrm{Li} *(2 p) \rightarrow \mathrm{Li}(2 s) .
\end{aligned}
$$

In this scenario, the observed distribution of emitting neutrals will reflect the degree to which the overall $\mathrm{Li}^{+}$and $\mathrm{e}$ (or $\mathrm{F}^{-}$) distributions overlap in space and time. Here we use time-gated i-CCD imaging, in conjunction with narrow bandpass interference filters that enable selection of specific transitions (and thus emitting species), to gain some insight into the production and evolution of selected excited species in the ablation plumes formed in both fs and ns PLA. As commented previously, Li b emission can be reabsorbed by ground state $\mathrm{Li}$ atoms (exciting them back to the $2 p$ state), whereas the $\mathrm{Li}$ a emission connects two excited states, neither of which are expected to have large steady state populations. Comparing time-gated images of the $\mathrm{Li}$ a and $\mathrm{Li} \mathrm{b}$ emissions thus allows estimation of the extent of radiation trapping and thus some insight into the evolving distribution of ground state $\mathrm{Li}$ atoms in the plume.

Figure 5 shows a series of wavelength filtered CCD images (viewed along $y$ ) of the plume accompanying ns PLA of LiF. The images were all collected using a $20 \mathrm{~ns}$ time gate but at different delays: $t=120 \mathrm{~ns}$ for images (a)-(d), $t$ $=520 \mathrm{~ns}$ for (e)-(h), and $t=800 \mathrm{~ns}$ for (i)-(l). Images (a), (e), and (i) (the top row) are unfiltered images, whereas (b), (f), and (j) (second row) are taken through a $610 \mathrm{~nm}$ narrow pass filter that transmits just the $\mathrm{Li}$ a emission, (c), (g), and (k) (third row) with a $670 \mathrm{~nm}$ narrow pass filter so as to select the Li b emission, and (d), (h), and (l) (bottom row) using a long pass filter that only transmits wavelengths $>780 \mathrm{~nm}$, thereby sampling predominantly the $812.4 \mathrm{~nm} \mathrm{Li} \mathrm{I} \mathrm{emission.}$ The white arrow in (a) indicates the axis of laser irradiation, with the beam incident at an angle of $45^{\circ}$ to the surface normal. The images were averaged over multiple laser shots. The intensity measured at each pixel of the CCD is stored to allow later profiling of the intensity distribution, but the dis- 


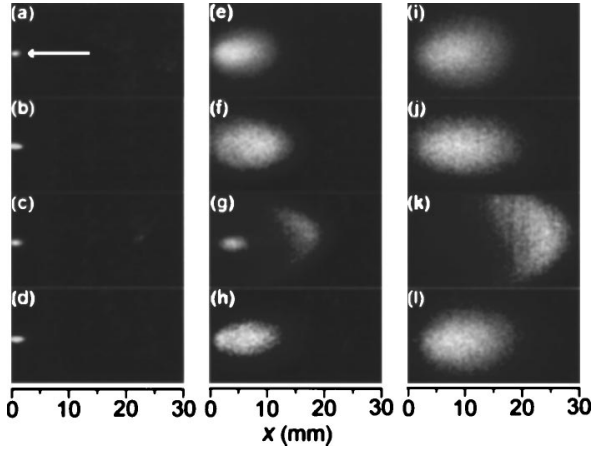

FIG. 5. Wavelength filtered CCD images of the plume accompanying ns PLA of LiF at $248 \mathrm{~nm}$. All images were collected with a $20 \mathrm{~ns}$ gate but with a variable delay of (a)-(d) $120 \mathrm{ns,} \mathrm{(e)-(h)} 520 \mathrm{~ns}$ and (i)-(1) $800 \mathrm{~ns}$. Images (a), (e) and (i) are unfiltered images, whereas (b), (f) and (j) were taken using a $610 \mathrm{~nm}$ narrow band pass filter, (c), (g) and (k) with a $670 \mathrm{~nm}$ narrow bandpass filter, and (d), (h) and (l) were taken using a filter that transmits light with wavelengths $>780 \mathrm{~nm}$. The white arrow in (a) indicates the axis of laser irradiation. The images were averaged over multiple laser shots and have been scaled to allow use of the entire gray-scale range.

played images have been scaled to allow use of the entire gray-scale range and to compensate for the decaying peak emission intensity. Comparing Figs. 5(i), 5(j), and 5(1), it is clear that the distribution and evolution of the total OES signal mirrors that of the selected images of $\mathrm{Li}$ a and the $>780 \mathrm{~nm}$ (mainly $812.6 \mathrm{~nm} \mathrm{Li} \mathrm{I)} \mathrm{emissions.} \mathrm{Such} \mathrm{a} \mathrm{finding}$ is consistent with the spatially resolved wavelength dispersed OES spectra shown in Fig. 3, which are dominated by the $\mathrm{Li}$ a peak. The $\mathrm{Li} \mathrm{b}$ emission shows a strikingly different time evolution, however, which can be described by studying a series of images recorded at progressively longer times. At early time, the Li b emission appears to peak at an $x$ value behind the maximum of the $\mathrm{Li}$ a image. At $t \sim 520 \mathrm{~ns}$, the distribution shows a double maximum along the $x$ axis [see Fig. 5(g)]. The first is closer to the laser-target interaction region than the peak of the $\mathrm{Li}$ a emission, the second more distant. The relative intensities of the leading (i.e., further from the ablation spot) and trailing maxima evolve with increasing $t$, so that by $t \sim 800 \mathrm{~ns}$ the $\mathrm{Li} \mathrm{b}$ emission profile again shows a single maximum that is significantly further forward than the peak in the $\mathrm{Li}$ a emission profile recorded at the same $t$. The observed time dependence of the Li b emission profile provides further illustration of the dramatic effects of self-absorption. As we show later, comparisons of the corresponding $\mathrm{Li} \mathrm{a}$ and $\mathrm{Li} \mathrm{b}$ images allow estimation of the time and spatial distribution of the ground state $\mathrm{Li}$ atoms.

A similar set of images was obtained following fs PLA of LiF. A selection of these are shown in Fig. 6. Three images are shown in each horizontal row of this figure. Each was recorded using a $20 \mathrm{~ns}$ collection gate which was delayed by, respectively, $t=120,280$, and $460 \mathrm{~ns}$. Images displayed in the top row are of the total emission, while those in the second and third rows were observed through, respectively, 610 and $670 \mathrm{~nm}$ narrow bandpass filters. Consistent with Fig. 3(b), the time-gated image of the total OES signal is found to be approximated well by a weighted sum of the $\mathrm{Li}$ $\mathrm{a}$ and $\mathrm{Li} \mathrm{b}$ distributions, with the latter making the greater contribution. Such an observation contrasts with that found in the case of ns PLA. The time evolution of the Li b emis-

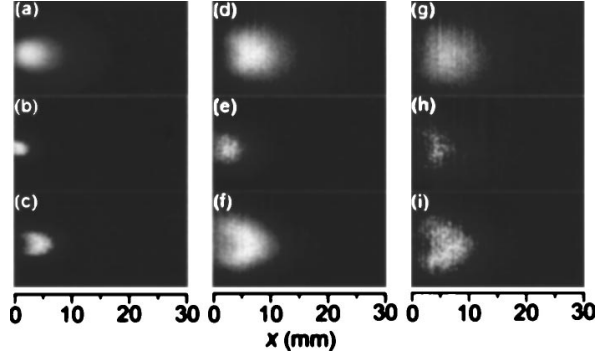

FIG. 6. Wavelength filtered CCD images of the plume accompanying fs PLA of LiF at $248 \mathrm{~nm}$. As in Fig. 6, all images were collected with a $20 \mathrm{~ns}$ gate but with variable delays of (a)-(c) $120 \mathrm{~ns}$, (d)-(f) $280 \mathrm{~ns}$ and (g)-(i) 460 ns. Images (a), (d) and (g) are unfiltered images, whereas (b), (e) and (h) were taken using a $610 \mathrm{~nm}$ narrow pass filter and (c), (f) and (i) with a $670 \mathrm{~nm}$ narrow pass filter. The images were averaged over multiple laser shots and have been scaled to use the entire gray-scale range.

sion profile appears less complicated in the case of fs PLA; at all $t$, the observed $\mathrm{Li} \mathrm{b}$ emission profile peaks in front of that of the $\mathrm{Li}$ a profile. Comparing images displayed in the first columns of Figs. 5 and 6 (both of which sets were taken at $t=120 \mathrm{~ns}$ ), we see that the emitting region of the plume appears larger in the case of fs PLA, implying a greater expansion velocity for material ejected when ablating with the shorter pulse duration.

As previously, ${ }^{27}$ estimates of the mean velocities $\langle v\rangle$ of the emitting components in the plume could be derived as follows. The emission intensity profiles along the $x$ axis of each of the captured time-gated CCD images were first analyzed, and the peak (or peaks) found by fitting with suitable mathematical functions, the exact nature of which was profile dependent and were merely used to obtain empirical fits. Figure 7 shows illustrative cuts from images of the filtered $\mathrm{Li}$ $\mathrm{b}$ emission recorded at $t=200$ and $600 \mathrm{~ns}$ for the case of (a) fs and (b) ns PLA of LiF. The double maximum in the Li b

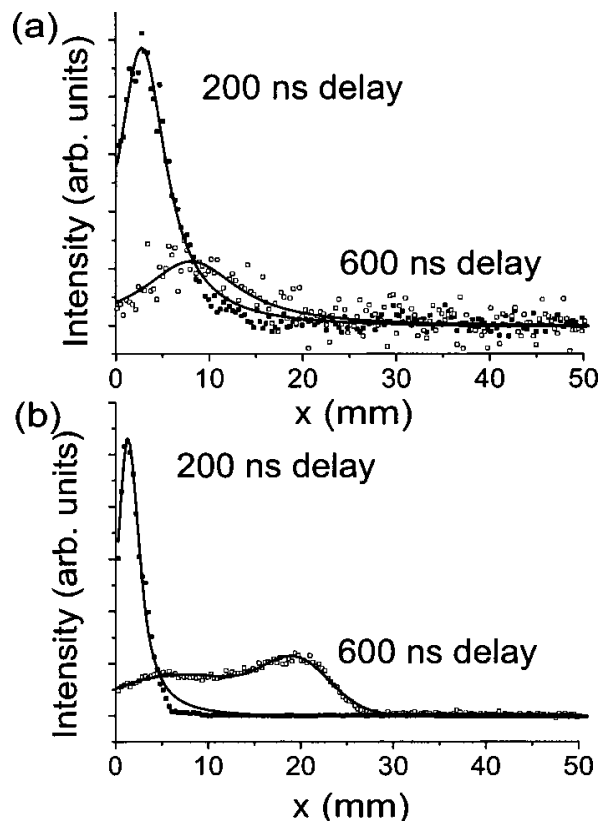

FIG. 7. Intensity $I$ vs $x$ profiles obtained from time-gated CCD images such as those shown in Figs. 5 and 6. (a) Two $I$ vs $x$ profiles of the Li b $(670.8 \mathrm{~nm})$ emission from fs PLA, at $t=200$ and $600 \mathrm{~ns}$. (b) Corresponding $I$ vs $x$ profiles from ns PLA of LiF at $248 \mathrm{~nm}$. 


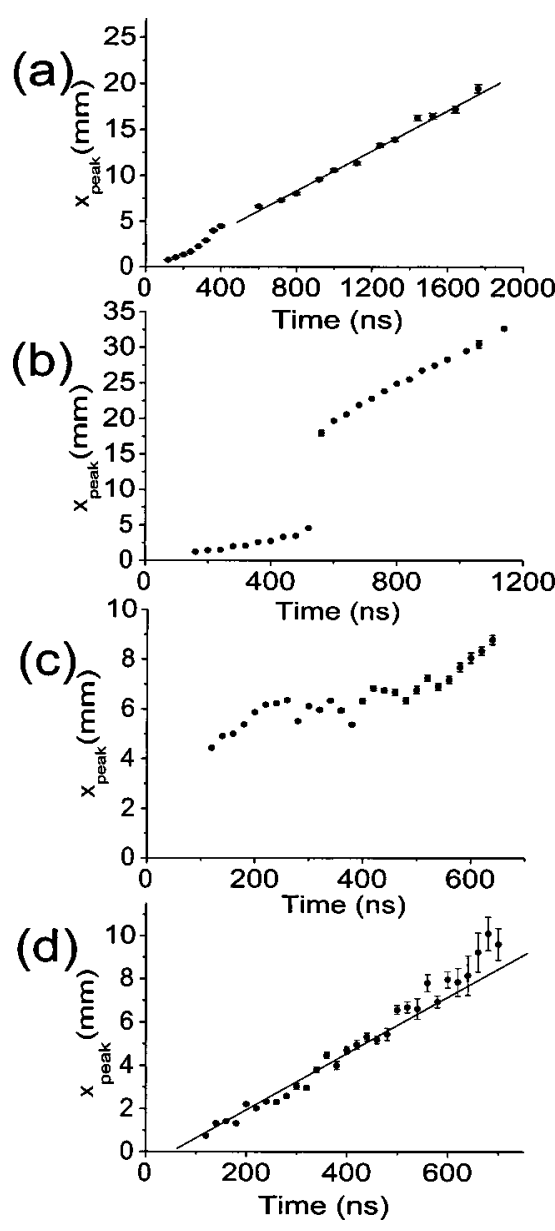

FIG. 8. Plots of $x_{\text {peak }}$ vs $t$ for the two Li I emissions following (a) ns PLA, filtered at $610 \mathrm{~nm}$, (b) ns PLA filtered at $670 \mathrm{~nm}$, (c) fs PLA filtered at $610 \mathrm{~nm}$, and (d) fs PLA filtered at $670 \mathrm{~nm}$. The gradients of the best-fit lines in (a) and (c) give respective mean velocities of $10.9 \pm 0.4 \mathrm{~km} / \mathrm{s}$ and $13 \pm 0.5 \mathrm{~km} / \mathrm{s}$.

emission accompanying ns PLA is clearly evident in the $t$ $=600 \mathrm{~ns}$ profile in Fig. 7(b). After fitting all of the profiles, the derived peak positions $x_{\text {peak }}$ were plotted as a function of time delay $t$ for both the $\mathrm{Li} \mathrm{a}$ and $\mathrm{Li} \mathrm{b}$ emissions, from both ns and fs PLA. Results for the Li a emission arising in the ns PLA, shown in Fig. 8(a), fit to a reasonable straight line, the gradient of which implies a value of $\langle v\rangle=10.9 \pm 0.4 \mathrm{~km} / \mathrm{s}$ for the emitting species. Figure 8(b) shows the corresponding plot for the Li b $(670 \mathrm{~nm})$ emission arising in the ns PLA of $\mathrm{LiF}$. The Li b intensity profiles generally show two maxima, the relative intensities of which are seen to swap over at $t$ $\sim 600 \mathrm{~ns}$. Only the $x_{\text {peak }}$ value of the predominant peak is plotted in the $x_{\text {peak }}$ versus $t$ plot displayed in Fig. 8(b)hence the discontinuity around $t \sim 600 \mathrm{~ns}$. The gradient of the early time part of this plot gives $\langle v\rangle=7.1 \pm 0.1 \mathrm{~km} / \mathrm{s}$, while the later part gives a much faster value $\langle v\rangle$ $=24.1 \pm 0.5 \mathrm{~km} / \mathrm{s}$. The corresponding $x_{\text {peak }}$ vs $t$ plots for the $\mathrm{Li}$ a and Li b emissions accompanying fs PLA of LiF are shown in Figs. 8(c) and 8(d). The former gives $\langle v\rangle$ $=13 \pm 0.5 \mathrm{~km} / \mathrm{s}$, while the latter plot shows some similarity with the later part of the equivalent ns ablation plot [Fig. 8(b)]. We note that the distance axis intercept of a line drawn through this data will miss the origin by a significant amount indicating that, as with the equivalent ns data, the effect of self-absorption on the emission profile cannot be ignored.

\section{DISCUSSION}

The optical emissions accompanying $248 \mathrm{~nm}$ PLA of $\mathrm{LiF}$, and the conclusions that might be drawn there from, are seen to be sensitively dependent both on the laser pulse duration and the particular transition chosen for monitoring. In addition, we find that the choice of pulse duration also leads to dramatic differences in the number and size of droplets produced by hydrodynamic sputtering. We now consider what insights these observations provide regarding the prevailing ablation mechanisms, and their dependence on the pulse duration.

The observed optical emission from both ns and fs PLA is dominated by transitions associated with neutral $\mathrm{Li}$. We postulate that these emissions arise mainly as a result of neutralization of $\mathrm{Li}^{+}$ions, by EIR, possibly supplemented by charge transfer from $\mathrm{F}^{-}$ions. Thus we must consider sources of $\mathrm{Li}^{+}$ions, for both $\mathrm{ns}$ and fs pulses, and the availability of electrons to take part in the EIR process. Likely sources of $\mathrm{Li}^{+}$ions in the PLA of $\mathrm{LiF}$ include direct ejection of ions by photon stimulated desorption (PSD) and the absorption of laser radiation [e.g., via inverse brehmsstrahlung excitation (IBE)] within the ablation plume, leading to heating, excitation, and ionization of the plume constituents.

The PSD of $\mathrm{Li}^{+}$ions from LiF single crystals has been investigated previously, using both $\mathrm{ns}^{20,21}$ and $\mathrm{fs}^{21}$ laser pulses. $\mathrm{Li}^{+}$photodesorbed from $\mathrm{LiF}$ surfaces was observed in both cases, even at subablation threshold fluences. Joly, Beck, and $\mathrm{Hess}^{21}$ determined average kinetic energies (KEs) of $\sim 5 \mathrm{eV}$ and $\sim 10 \mathrm{eV}$ for $\mathrm{Li}^{+}$ions desorbed following $266 \mathrm{~nm}$ excitation of $\mathrm{LiF}$ using, respectively, $\mathrm{ns}$ and fs laser pulses. These authors also determined that the energy thresholds and power dependences for $\mathrm{Li}^{+}$ion formation were similar in the two cases, notwithstanding the expectation that different excitation mechanisms would likely dominate when using such different laser pulses durations. Bandis, Langford, and Dickinson ${ }^{20}$ reported a similar KE $(6.7 \mathrm{eV})$ for $\mathrm{Li}^{+}$ions desorbed in the ns pulsed laser excitation of LiF at $248 \mathrm{~nm}$, and noted that this KE value (and those of the cations from other alkali and alkaline earth halide crystals) matched well with the predictions of a model which assumed that the electrostatic energy of the ion prior to expulsion is converted into $\mathrm{KE}$, with little partitioned into inelastic processes such as lattice distortions or phonon emission. PSD can also be expected to produce a significant amount of neutral material along with the ionic component - to which, of course, the ion time-of-flight (TOF) experiments are insensitive. If there is a thermal component to this PSD derived source of neutral material it will surely be more important in the case of ns PLA since, under fs excitation, irradiation will have finished before phonon-phonon coupling has the chance to cause much increase in the temperature of the lattice.

The relative importance of laser-plume interactions is also heavily dependent upon the laser pulse duration, becoming increasingly significant as the laser pulse duration increases. The ionization potential of $\mathrm{Li}(5.4 \mathrm{eV})$ is much 
lower than that of $\mathrm{F}(17.4 \mathrm{eV})$. The $\mathrm{KrF}$ photon energy is $5.0 \mathrm{eV}$. Such photons thus provide enough energy to ionize all excited states of the $\mathrm{Li}$ atom. In addition, we note that the $6 p \leftarrow 2 s$ absorptions of Li fall within the $\mathrm{KrF}$ laser bandwidth, thus offering a facile route to resonance enhanced two photon ionization of ground state $\mathrm{Li}$ atoms. In contrast, a ground state $\mathrm{F}$ atom would have to absorb at least $4 \mathrm{KrF}$ photons to be ionized. $\mathrm{Li}$ atoms are thus by far the most readily ionizable species within the plume.

Given these background considerations, we propose that laser-plume interactions will be rather minor in the case of fs PLA and that much of the $\mathrm{Li}^{+}$ion yield is produced by direct laser-target interactions, such as PSD. The plasma-annealing model $^{28}$ has been applied to explain the highly ionized nature of materials irradiated with ultrashort laser pulses. In this model, the high levels of excitation produced by the laser promote a significant proportion of the electrons into excited states, rendering the lattice unbound and transforming material in the laser interaction volume into a high-density plasma. Note that if purely fast thermal evaporation were occurring during fs irradiation, little or no ionic material would be expected, as the ejection temperatures (typically $<1 \mathrm{eV}$ ) are significantly lower than the relevant ionization potentials. The model prediction that the ratio of the ion to neutral desorption yields will be much higher in the case of fs PLA is born out experimentally, in as much that $\mathrm{F}^{*}$ emission (which we attribute to $\mathrm{F}^{+}+\mathrm{e} \rightarrow \mathrm{F}^{*}$ ) was only observed in the fs PLA of LiF (see Fig. 4). The $x_{\text {peak }}$ vs $t$ plot of the Li a emission accompanying fs PLA of LiF [Figure 8(c)] has a gradient $\langle v\rangle=13 \pm 0.5 \mathrm{~km} / \mathrm{s}$, equivalent to a KE of $\sim 6.1 \mathrm{eV}$. Emission at this wavelength will be much less sensitive to the effects of any radiation trapping than the $\mathrm{Li} \mathrm{b}$ emission. Such plots should thus provide a reasonable measure of the velocity of the emitting $\mathrm{Li}^{*}$ atoms in the plume. The similarity between this KE value and that obtained by Joly, Beck, and $\mathrm{Hess}^{21}$ from TOF mass spectroscopy lends further support to the proposal that emitting $\mathrm{Li}^{*}$ atoms can be a useful surrogate for their precursor $\mathrm{Li}^{+}$ions. We recognize that the fluences used in the present work are significantly higher than those employed by Joly and co-workers, but also note that these authors reported a small shift to earlier arrival time (i.e., higher KE) as the fluence was reduced. Such an increase in KE with reduced fluence is of course the opposite to that which might be expected if either surface or space charge effects were controlling the velocity distribution. As discussed previously, ${ }^{27}$ there are also a number of reasons why analyses of the OES of excited neutrals of the kind reported here are likely to underestimate the true $\langle v\rangle$ of nascent ions (even in the limit that EIR is entirely responsible for the emitting species). One obvious limitation is that the measured images are squashed two-dimensional projections of a three-dimensional cloud of emitting particles. We analyze a slice along $x$, but not all emitting particles fly along the surface normal; the velocity of any emitting particles with some component along $y$ will be underestimated. Further, true proportionality between $\mathrm{Li}^{+}$and $\mathrm{Li}^{*}$ would require an EIR efficiency that is independent of $x$. In practice, such collision-induced processes are likely to be less efficient in less dense (i.e., peripheral) regions of the plume.
During a ns pulse the temperature of the irradiated region of the target will increase rapidly until it boils. The larger size and much higher density of "splashed" droplets observed in the films deposited by ns PLA suggests that the target surface may have spent some significant time in a molten state prior to material boiloff. If $\mathrm{Li}$ atoms were to boiloff the target surface at the boiling point $\left(T_{\text {boil }}\right)$ of $\mathrm{LiF}$ $\left(\sim 1676{ }^{\circ} \mathrm{C}\right.$ ), the resulting $\mathrm{Li}$ atoms (of mass $m$ ) would have a mean speed $\left(8 \mathrm{k} T_{\text {boil }} / \pi m\right)^{\frac{1}{2}}=2.44 \mathrm{~km} / \mathrm{s}$. Some fraction of these $\mathrm{Li}$ atoms will be ionized by laser-plume interactions. Thus the distribution of $\mathrm{Li}^{+}$ions resulting from ns PLA of $\mathrm{LiF}$ is likely to be a fusion of two components both of which require the presence of the laser radiation: one generated directly by PSD, the second from laser-plume interactions, which are then mediated by Coulombic interactions between the electrons and ions. Contributions from PSD will likely maximize early in the laser pulse. Laser-plume interactions will increase as the plume density increases and, in extreme cases, may attenuate the laser intensity reaching the target substantially. The mean velocity for $\mathrm{Li}^{+}$ions we deduce from analysis of the Li a OES is $\langle v\rangle=10.9 \pm 0.4 \mathrm{~km} / \mathrm{s}$ [see Fig. $8(\mathrm{a})]$ equivalent to a $\mathrm{KE}$ of $\sim 4.3 \mathrm{eV}$. Similar analysis of the ns plume images obtained using the $>780 \mathrm{~nm}$ filter gives $\langle v\rangle=11.5 \pm 0.4 \mathrm{~km} / \mathrm{s}$ - consistent with the view that the Li I emission at $812.6 \mathrm{~nm}$ is the dominant contributor to this long wavelength emission. Both estimates of the $\mathrm{Li}^{+}$ion velocity are comparable to, but somewhat lower than, the value deduced above for the case of fs PLA of LiF. By way of comparison, Bandis ${ }^{20}$ deduced a mean $\mathrm{KE} \sim 6 \mathrm{eV}$ (and a full width half maximum $\sim 3 \mathrm{eV}$ ) for the photodesorbed $\mathrm{Li}^{+}$ions produced in the subthreshold ns irradiation of LiF, while Joly $^{21}$ obtained a mean KE value of $5 \mathrm{eV}$-albeit at incident fluences significantly lower than those used in the current work.

We now consider the distributions of ground state material in the plume. The plume images filtered so as to select the $\mathrm{Li} \mathrm{a}(3 d \rightarrow 2 p)$ and $\mathrm{Li} \mathrm{b}(2 p \rightarrow 2 s)$ emissions are dramatically different, particularly in the case of ns PLA. Given the short radiative lifetimes of the $3 d$ and $2 p$ levels (relative to the observation times), we can envisage no reason why the true distribution of $\mathrm{Li}^{*}(3 d)$ or $\mathrm{Li}^{*}(3 s)$ atoms should differ significantly from that for $\mathrm{Li}^{*}(2 p)$. Hence the conclusion that the differences between the respective emission distributions must be a consequence of reabsorption of photons produced by Li b decay by a high density of ground state $\mathrm{Li}$ atoms. The $\mathrm{Li}$ a transition, in contrast, terminates on an excited state $(2 p)$, the steady state number density of which will be much lower than that of the ground state. Its intensity profile should thus be much less affected by radiation trapping. Differences between the $\mathrm{Li}$ a and $\mathrm{Li}$ b images recorded at any given $t$ might thus provide some insight into the distribution of ground state $\mathrm{Li}$ atoms at that time. This was achieved by rescaling the intensity $(I)$ vs $x$ distribution derived from each Li $\mathrm{b}$ image so that it matched the large $x$ part of the corresponding $\mathrm{Li}$ a distribution, on the basis that this is a region where the density of ground state Li atoms will be lowest, and the effect of self-absorption of the $\mathrm{Li}$ b emission least important. A first estimate of the attenuation profile was 

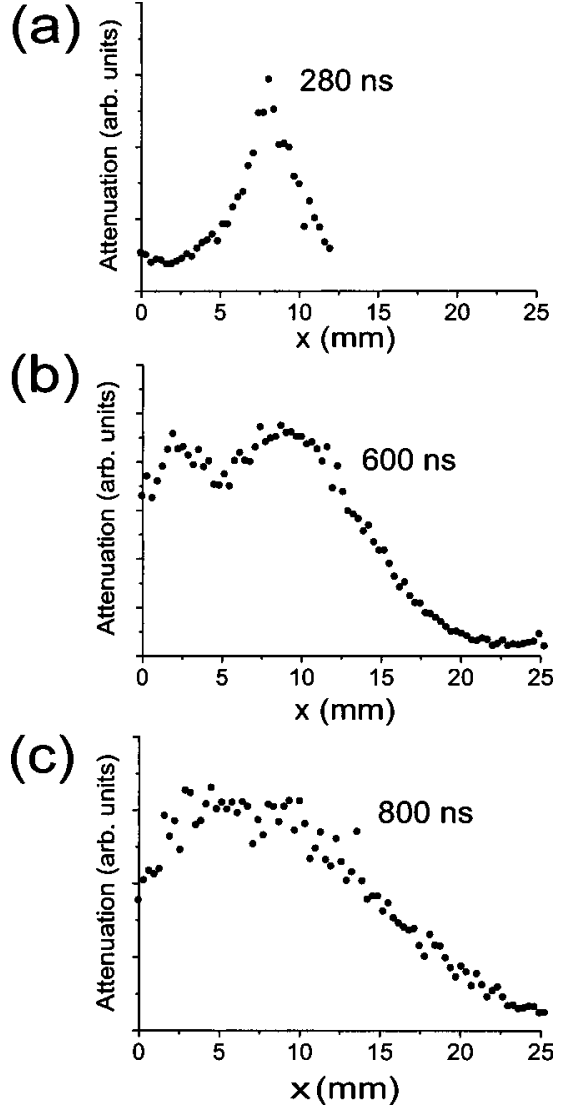

FIG. 9. Attenuation profiles obtained by dividing the (suitably rescaled) $I$ vs $x$ distribution of the $\mathrm{Li}$ a emission by that of the $\mathrm{Li} \mathrm{b}$ emission recorded at $t=280 \mathrm{~ns}$ (a), $600 \mathrm{~ns}$ (b) and $800 \mathrm{~ns}$ (c).

then obtained by dividing the $I$ vs $x$ distribution of the $\mathrm{Li}$ a emission by that of the $\mathrm{Li} \mathrm{b}$ emission. This process was repeated for the range of CCD images taken at different $x$ following ns PLA of LiF. Figure 9 shows three attenuation profiles so derived. The $t=280 \mathrm{~ns}$ profile [Fig. 9(a)] is quite sharp, and peaks ahead of $x_{\text {peak }}$ for the corresponding $\mathrm{Li}$ a emission. By $t=600 \mathrm{~ns}$ [Fig. 9(b)] the attenuation profile is much broader and shows a hint of bimodality, and by $t$ $=800 \mathrm{~ns}$ the profile is broader still, and appears to peak at smaller $x$ than the $x_{\text {peak }}$ value observed at $600 \mathrm{~ns}$.

As commented previously, we can envisage two limiting mechanisms that may contribute to the distribution of ejected material-one, PSD, essentially electronic in origin, the other thermal. We anticipate that the "zero-order" kinetic energy distributions of the ablated $\mathrm{Li}$ (and F) atoms and ions arising via the two mechanisms will be different, but that the two distributions will fuse with increasing $t$. Given the evidence of molten particulates in the deposited films, we can also anticipate a major thermal contribution to the ablation plume. It is hard to reconcile such a picture with the apparently narrow, "fast" distribution of ground state Li atoms implied by the $t=280 \mathrm{~ns}$ attenuation profile. The long time $(t=800 \mathrm{~ns})$ attenuation profile is much closer to the shifted Maxwellian velocity distribution that is characteristic of most ablation plumes. ${ }^{3}$

Figure 10 shows a plot of the centers of gravity $(\langle x\rangle)$ of the deduced attenuation profiles as a function of $t$. Such a plot of propagation distance versus time should pass through

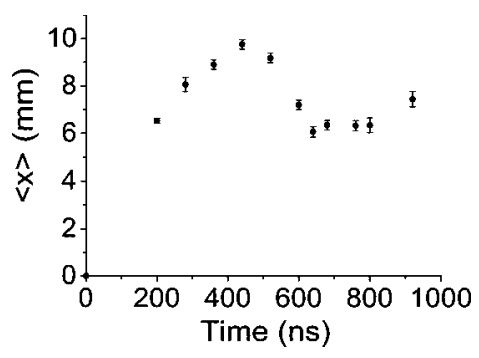

FIG. 10. Plot of the centers of gravity $(\langle x\rangle)$ of the deduced attenuation profiles of the Li b emission as a function of $t$.

the origin; the long time measurements thus correspond to a mean propagation velocity $\langle v\rangle \sim 8.5 \mathrm{~km} / \mathrm{s}$ but, clearly, the deduced $\langle x\rangle$ values at early $t$ appear to be too large. This apparent deviation from the (expected) constant expansion velocity, and the seemingly unphysical nature of the ground state velocity distribution implied by the attenuation profiles derived at short $t$, both highlight a shortcoming of the preceding analysis - namely the assumption that the Li a emission is immune to the effects of self-absorption. Radiation trapping will be most important in the densest regions of the plume, i.e., at early $t$, before the ablated material has expanded into an ever increasing volume. The self-absorption considered thus far is of Li b emission, but the net effect of the emission-reabsorption cycle is to maintain an excessive (relative to local thermal equilibrium) population in the $2 p$ state which will serve to attenuate the $3 d \rightarrow 2 p$ and $3 s \rightarrow 2 p$ emissions in the densest regions of the plume. The effect of such reabsorption is imperceptible in the $t=800 \mathrm{~ns}$ profile [Fig. 9(c)] - where the plume density is lowest, but is responsible for the central dip in the $t=600 \mathrm{~ns}$ profile [Fig. 9(b)] and the apparent dramatic loss of "attenuation" (and thus the implied absence of ground state Li atoms) at small $x$ in the $t=280 \mathrm{~ns}$ profile [Fig. 9(a)]. Thus, the sharp peak in the deduced attenuation profile is a consequence of the method used to estimate the attenuation. At small $t$, both the $\mathrm{Li}$ a and $\mathrm{Li}$ b signals are affected by reabsorption, so their ratio no longer represents simply the attenuation by ground state $\mathrm{Li}$ atoms. It should be noted that the collected OES intensity at a point on the CCD is a summation of all the emission produced along a line perpendicular to the $x$ axis. For this reason it would be difficult to obtain an numerical estimate of the density of absorbing material, without previously knowing what the intensity would be without the effect of attenuation, and how the plume density varies along the line of sight.

The observed attenuation is significantly less in the fs ablation experiments, indicating that the density of ground state Li atoms was lower in the case of fs PLA. This must be primarily due to the general reduction in the amount of ablated material (as the total energy delivered is less for the fs pulses), supplemented by a reduction in the relative yield of ground state neutrals. The reduction in the number of droplets, along with the more intense $\mathrm{F}$ emission detected in the plume, is evidence for characterizing the fs ablation as explosive boiling rather than thermal sputtering. The low melting point and high thermal expansion coefficient of $\mathrm{LiF}$ would mean that significant hydrodynamic sputtering would 
be expected if the target surface underwent transient melting, as is observed for ns ablation. For ns ablation at $193 \mathrm{~nm}$, melting of the target surface was clearly observed. ${ }^{10}$ The OES images following fs ablation at $248 \mathrm{~nm}$, filtered to show the Li $\mathrm{b}$ emission, show evidence for some radiation trapping. Comparing Figs. 6(b) and 6(c) or Figs. 6(e) and 6(f), it is observed that the region of most obvious attenuation of the $\mathrm{Li} \mathrm{b}$ emission always occurs at the same position as the peak in the $\mathrm{Li}$ a images. The evolution of this attenuation profile (not shown here) is much simpler for the fs images, with no evidence for any reabsorption of the $\mathrm{Li}$ a peak at small $t$. This serves to reinforce the view that the density of ground state material in the plume is significantly lower in the case of fs PLA, and that re-population of $\operatorname{Li}(2 p)$ as a result of radiation trapping is much less important in this case. Since IBE within the plume will be relatively much less important in the case of fs PLA, the $\mathrm{Li}^{+}$ions invoked to account for the observed optical transitions must be formed during the initial laser-target interaction. Thus we should anticipate a much reduced contribution from any slower, thermally evolved component, and a relatively higher $\langle v\rangle$ for the $\mathrm{Li}^{+}$ions in the plume (and thus for the imaged $\mathrm{Li}^{*}$ atoms) than in the case of ns PLA. Such expectations accord with experimental observation.

\section{CONCLUSION}

PLA of LiF using fs pulses at $248 \mathrm{~nm}$ was found to produce a plume with a higher proportion of energetic/ electrically excited components, including excited $\mathrm{F}$ atoms and ions, than that from ns PLA of the same material at the same wavelength. However, the amount of target material removed per shot was significantly less. Ns ablation produces a plume with a much higher density of ground state $\mathrm{Li}$ atoms, as revealed by the evident reabsorption of $\operatorname{Li}(2 p$ $\rightarrow 2 s$ ) optical emission. Films grown by fs PLA, especially those deposited on substrates mounted in an off-axis geometry, are contaminated with fewer (and smaller) droplets. Such observations imply that fs PLA of LiF exhibits characteristics of explosive boiling, whereas ns ablation is dominated by thermal evaporation of the transiently heated target surface.

Optical emission measurements are often used to provide an estimate of the expansion velocity of material in ablation plumes. The present work highlights some of the potential pitfalls associated with such measurements, most obviously when the monitored emission involves transition to a state [here the ground $(2 s)$ state] that is present in high number density. Radiation trapping in such cases can result in very misleading spatial and temporal emission profiles. Comparisons of time-gated spatially resolved images of the $\operatorname{Li}(3 d \rightarrow 2 p)$ and $\operatorname{Li}(2 p \rightarrow 2 s)$ emissions arising in the ns PLA of $\mathrm{LiF}$ enabled estimation of the distribution of ground state $\mathrm{Li}$ atoms in the plume. Indeed, more thorough analysis of these images revealed that the density of $\operatorname{Li}(2 p)$ atoms in the early stages of the plume expansion was sufficiently high (as a result of $\operatorname{Li}(2 p \leftarrow 2 s)$ reabsorption) that even the $\operatorname{Li}(3 d$ $\rightarrow 2 p$ ) emission profiles accompanying ns PLA of LiF show evidence of radiation trapping. These observations serve to reinforce the view that the plume arising in the ns PLA of $\mathrm{LiF}$ contains a much higher density of ground state material than that formed using fs pulses.

\section{ACKNOWLEDGMENTS}

The authors thank Demetrios Anglos and Costas Fotakis for their assistance with this project and S.J.H. and G.M.F. would like to thank A. Egglezis and A. Klini for helping with the laser and vacuum systems, respectively. The authors are grateful to EPSRC for funding via the portfolio grant LASER and the Carbon Based Electronics programme. Experiments were carried out at the Ultraviolet Laser Facility operating at IESL FORTH and supported by the EU through the Research Infrastructures activity of FP6 (Project: LaserlabEurope Contract No: RII3-CT-2003-506350.)

${ }^{1}$ Pulsed Laser Deposition of Thin Films edited by D. B. Chrisey and G. K. Hubler (Wiley, New York, 1994), and references therein.

${ }^{2}$ S. Georgiou and F. Hillenkamp, Chem. Rev. (Washington, D.C.), 103 (2003), and references therein.

${ }^{3}$ M. N. R. Ashfold, F. Claeyssens, G. Fuge, and S. J. Henley, Chem. Soc. Rev. 33, 23 (2004), and references therein.

${ }^{4}$ R. Schlaf, B. A. Parkinson, P. A. Lee, K. W. Nebesny, G. Jabbor, B. Kippelen, N. Peyghambarian, and N. R. Armstrong, J. Appl. Phys. 84, 6729 (1998)

${ }^{5}$ Y. M. Wang, K. W. Wong, S. T. Lee, M. Nishitani-Gamo, I. Sakaguchi, K. P. Loh, and T. Ando, Diamond Relat. Mater. 9, 1582 (2000).

${ }^{6}$ Y. Harada and S. Hashimoto, Phys. Rev. B 68, 045421 (2003).

${ }^{7}$ L. S. Hung, C. W. Tang, and M. G. Mason, Appl. Phys. Lett. 70, 152 (1997).

${ }^{8}$ T. M. Brown, I. S. Millard, D. J. Lacey, J. H. Burroughes, R. H. Friend, and F. Cacialli, Synth. Met. 124, 15 (2001).

${ }^{9}$ A. Perea, J. Gonzalo, C. N. Afonso, S. Martelli, and R. M. Montereali, Appl. Surf. Sci. 138-139, 533 (1999).

${ }^{10}$ S. J. Henley, M. N. R. Ashfold, and S. R. J. Pearce, Appl. Surf. Sci. 217, 68 (2003).

${ }^{11}$ Handbook of Infrared Optical Materials, edited by P. Klocek (Dekker, New York, 1991).

${ }^{12}$ F. J. Himpsel, L. J. Terminello, D. A. Lapiano-Smith, E. A. Eklund, and J. J. Barton, Phys. Rev. Lett. 68, 3611 (1992).

${ }^{13}$ R. Kelly and A. Miotello, in Pulsed Laser Deposition of Thin Films, edited by D. B. Chrisey and G. K. Hubler (Wiley, New York, 1994), pp. 55-87.

${ }^{14}$ R. L. Webb, L. C. Jensen, S. C. Langford, and J. T. Dickinson, J. Appl. Phys. 74, 2338 (1993)

${ }^{15}$ N. Ichimura, H. Kondo, Y. Harada, and S. Hashimoto, J. Lumin. 87-89, $586(2000)$.

${ }^{16}$ P. Dyer, S. Waswadi, and C. Walton, Appl. Phys. A: Mater. Sci. Process. 76, 817 (2003).

${ }^{17}$ M. Heynk, F. Costache, and J. Reif, Appl. Surf. Sci. 197, 90 (2002).

${ }^{18}$ M. Henyk, F. Costache, and J. Reif, Appl. Surf. Sci. 186, 381 (2002).

${ }^{19}$ M. Henyk and J. Reif, Appl. Surf. Sci. 208, 71 (2003).

${ }^{20}$ C. Bandis, S. C. Langford, and J. T. Dickinson, Appl. Phys. Lett. 76, 421 (2000).

${ }^{21}$ A. Joly, K. M. Beck, and W. P. Hess, Appl. Phys. A: Mater. Sci. Process. 69, S153 (1999).

${ }^{22}$ S. Szatmari and P. Schafer, Opt. Commun. 68, 196 (1988).

${ }^{23}$ S. Tzortzakis, B. Lamouroux, A. Chiron, S. Moustaizis, D. Anglos, M. Franco, B. Prade, and A. Mysyrowicz, Opt. Commun. 197, 131 (2001).

${ }^{24}$ WebElements, www.webelements.com

${ }^{25}$ Scion Image, www.scioncorp.com

${ }^{26}$ NIST Atomic Spectra Database, http://physics.nist.gov/cgi-bin/AtData/ main_asd

${ }^{27}$ F. Claeyssens, R. Lade, K. Rosser, and M. Ashfold, J. Appl. Phys. 89, 697 (2001).

${ }^{28}$ J. A. Van Vechten, R. Tsu, F. W. Saris, and D. Hoonhout, Phys. Lett. 74A, 417 (1979). 